\title{
Mística, yerbas santas y solidaridad: memoria del cuidado no médico de la lepra en Agua de Dios ${ }^{1}$
}

\author{
Mystique, Holy Herbs and Solidarity: Memory \\ of Non-Medical Care of Leprosy in Agua de Dios
}

Mística, ervas santas e solidariedade: memória do cuidado no médico da lepra em Agua de Dios

\author{
María Teresa Buitrago Echeverri, PhD ${ }^{2 *}$
}

Recibido: 30 de julio de 2017 - Aceptado: 20 de septiembre de 2018

Doi: http://dx.doi.org/10.12804/revistas.urosario.edu.co/revsalud/a.8121

Para citar este artículo: Buitrago-Echeverri MT. Mística, yerbas santas y solidaridad: memoria del cuidado no médico de la lepra en Agua de Dios. Rev Cienc Salud. 2019;17(especial):88-108. Doi: http://dx.doi.org/10.12804/revistas.urosario.edu.co/revsalud/a.8121

\section{Resumen}

Introducción: la representación social de la lepra como una enfermedad del pasado, el carácter local que la circunscribe a los sanatorios y el escaso contacto de trabajadores de la salud foráneos con la enfermedad hace pensar que el conocimiento sobre su cuidado ha circulado entre generaciones de una manera más o menos confinada. Ese saber que incorpora no solo conocimiento biomédico, sino expresiones en saberes, creencias, prácticas, relatos, los actores que las construyen y recrean y su relación compleja con las dinámicas de inserción en el territorio, se constituye hoy, en sí mismo, en un patrimonio vivo, que merece ser narrado. Desarrollo: se parte de comprender la memoria de los cuidados no médicos de la lepra en Agua de Dios, por medio de un estudio etnográfico, cuyos resultados permiten proponer dos expresiones. La primera involucra a agentes de salud no médicos y su relación con los sujetos de cuidado; que muestra que el cuidado demanda no solo conocimiento científico, sino también disposiciones particulares de los sujetos, como capacidad de escucha, observación, paciencia y trascendencia, acompañadas de un posicionamiento y negociación cotidianos en el territorio. La segunda está compuesta por las alternativas de cuidado intermediadas por otros agentes con saberes autorizados, que agrupan una amplia farmacopea relacionada con el uso de plantas medicinales, referencias a curanderos y prácticas solidarias de tutoría entre pacientes y relaciones de su bienestar con el medio ambiente. Conclusiones: todas estas expresiones de cuidado desafían los discursos y prácticas canónicas de la biomedicina alrededor de esta enfermedad.

Palabras clave: memoria, cuidados, lepra, saber autorizado, modelo médico hegemónico.

1 Los resultados de este trabajo fueron presentados en el simposio Salud y Sociedad del V Congreso Latinoamericano de Antropología en la ciudad de Bogotá, el 5 de junio de 2017.

2 Facultad de Enfermería, Pontificia Universidad Javeriana, Bogotá.

Autora de correspondencia: mtbuitragoe@gmail.com 


\section{Abstract}

Introduction: The social representation of leprosy as a disease from the past, its local character that circumscribes it to the sanatoria, and the scarce contact of health workers with it suggest that the knowledge about its care has circulated between generations more or less confined. This confined knowledge, which incorporates not only biomedical knowledge but also expressions in knowledge, beliefs, practices, stories, the actors that construct and recreate them and their complex relationship with the dynamics of insertion in the territory in a living heritage, deserves to be narrated. Development: We begin by understanding the memory of the non-medical care of leprosy in Agua de Dios, through an ethnographic study, whose results allow us to propose two expressions of these. The first one, involving the non-medical health agents (especially nurses) and its relationship with care subjects, showed that this demands particular dispositions from the subjects as listening ability, observation, patience, and transcendence, accompanied by daily positioning and negotiation in the territory. The second is the care alternatives mediated by other agents with authorized knowledge that group a wide pharmacopeia related to the use of medicinal plants; references to healers and solidarity practices of mentoring between patients and relationships of their well-being with the environment. Conclusions: All these expressions of care challenge the discourses and canonical practices of biomedicine around this disease.

Keywords: Memory, care, leprosy, know authorized, hegemonic medical model.

\section{Resumo}

Introdução: a representação social da lepra como uma doença do passado, seu carácter local que a circunscreve aos sanatórios, e o escasso contato de trabalhadores da saúde estrangeiros com a doença, faz pensar que o conhecimento sobre seu cuidado, tem circulado entre gerações de uma maneira mais ou menos confinada. Esse saber "confinado", que incorpora não só conhecimento biomédico, senão expressões em saberes, crenças, práticas, relatos, os atores que as constroem e recriam e sua relação complexa com as dinâmicas de inserção no território, constitui-se hoje em si mesmo, em um patrimônio vivo, que merece ser narrado. Desenvolvimento: partimos de compreender a memória dos cuidados não médicos da lepra em Agua de Dios, através de um estudo etnográfico, cujos resultados nos permitem propor duas expressões destes. A primeira que envolve agentes de saúde não médicos e sua relação com os sujeitos de cuidado, mostrou que esta demanda não só conhecimento "científico", mas também disposições particulares dos sujeitos como capacidade de escuta, observação, paciência e transcendência, acompanhadas de um posicionamento e negociação cotidianos no território. A segunda a constituem as alternativas de cuidado intermediadas por outros agentes com saberes "autorizados” que agrupam uma ampla farmacopeia relacionada com o uso de plantas medicinais; referências a curandeiros e práticas solidárias de tutoria entre pacientes e relações de seu bem-estar com o meio ambiente. Conclusões: todas estas expressões de cuidado, desafiam os discursos e práticas canónicas da biomedicina ao redor desta doença.

Palavras-chave: memória, cuidados, lepra, saber autorizado, Modelo médico hegemônico.

\section{Introducción}

I hecho de que la lepra haya sido manejada con medidas de carácter aislacionista y que
en el conocimiento médico hoy día se vea como una enfermedad casi erradicada, según 
los cánones epidemiológicos de la Organización Mundial de la Salud (oms), ha hecho de ella un tema de expertos y un olvido en la salud pública. Solo a finales de los noventa, se revive el interés epidemiológico internacional sobre esta y se insiste en profundizar en esfuerzos para erradicarla, que corresponde a tener prevalencias por debajo de 1 caso por cada 10000 habitantes. Sin embargo, este indicador minimiza la realidad de la aparición todos los años de casos de lepra en el mundo y en Colombia, registrándose en 2012 y 2014, 1282 casos, con un promedio de 427.3 casos por año (1). Esta situación disminuye, según los reportes para 2015 y 2016, con 390 y 345 casos, respectivamente, pero aumenta nuevamente en 2017, informando para la semana epidemiológica 52 un total de 452 casos, según el Instituto Nacional de Salud $(2,3)$.

La representación social de ser una enfermedad del pasado, su carácter local que la circunscribe a los sitios de ubicación geográfica de los sanatorios (antiguos lazaretos), además del escaso contacto de trabajadores de la salud foráneos con el mal, hace pensar que el conocimiento sobre el cuidado de la lepra ha circulado entre generaciones que han constituido los sanatorios y sus municipios como territorios sociales, de una manera más 0 menos confinada.

Con el objetivo de indagar por la memoria de cuidado de la lepra, y avanzar en su reconocimiento como parte del legado que el territorio de Agua de Dios tiene para compartir, se hizo un acercamiento a su conocimiento como parte de una investigación interdisciplinar más amplia denominada "Aproximación a la construcción territorial de Agua de Dios a partir de la lepra como hecho fundante". La importancia de ese trabajo radica en que este saber confinado sobre el cuidado de lepra, que incorpora no solo conocimiento biomédico, las expresiones en saberes, creencias, prácticas, relatos, los actores que las construyen y recrean y su relación compleja con las dinámicas de inserción en el territorio, se constituye hoy, en sí mismo, en un patrimonio vivo sobre el cuidado de la lepra que merece ser narrado y construido, como un elemento no solo de conocimiento y memoria, sino de resistencia y permanente negociación de saberes entre lógicas diversas de cómo se vive con la enfermedad.

En este estudio cualitativo de carácter etnográfico, la indagación comparte con Marshall y Rossman el ser una investigación asentada en la experiencia de los sujetos y en la comprensión que de la realidad social ellos hacen y, además, con Vasilachis, lo que establece como "un modo particular de acercamiento a la indagación: una forma de ver y una manera de conceptualizar una cosmovisión unida a una perspectiva teórica para comunicar e interpretar la realidad" (4-6).

Para el propósito de ahondar en el conocimiento de la memoria del cuidado no médico de la lepra y su comprensión e interpretación, en relación con la triangulación teórica necesaria, se tuvieron como base algunos referentes que aportan a esta tensión que se evidencia en los resultados, en tanto este tipo de cuidados subvierte los protocolos circunscritos por la biomedicina convencional, que solo reconoce como válido este tipo de saber. Aquí la propuesta de 
Menéndez sobre el modelo médico hegemónico resulta pertinente, al comprenderse como esa manera en que la biomedicina organiza su saber y su actuar de manera vertical e impone unas maneras válidas de acercarse al manejo de las enfermedades, desconociendo no solo otros saberes, sino, en muchas ocasiones, que el cuidado tiene que ver con personas y sus maneras intersubjetivas de estar en el mundo y no solo con patologías, úlceras o partes del cuerpo que curar (7).

De igual manera, fue importante analizar lo encontrado en relación con lo que la antropología plantea sobre la medicalización del quehacer de los trabajadores de la salud, no solo en las prácticas, sino en las maneras como se conceptualiza y decide sobre los cuerpos, y cómo las lógicas del cuidado no médico de la lepra, presentes en las narrativas de personal de salud y personas con lepra en Agua de Dios en la cotidianidad de sus prácticas, subvierten esta medicalización y reconocen la importancia de las transacciones de conocimientos e intereses que hacen parte del cuidado, y en especial cuando se trata de la lepra, por ser esta una enfermedad que produce secuelas que se cronifican (8-10).

En esa subversión de la medicalización de la que dieron cuenta las diversas expresiones de cuidado que desafían el conocimiento biomédico e instauran en las prácticas otros tipos de saberes, resultó pertinente traer a colación el concepto de saber autorizado, propuesto desde la antropología por Brigitte Jordan y desarrollado posteriormente por Sargent $(11,12)$. Este hace referencia no al conocimiento que se asocia con personas con posiciones de autoridad, en el caso de la lepra, el conocimiento de la biomedicina usado por el médico, sino al "conocimiento sobre el cual se toman decisiones sobre determinado asunto, en un grupo social” (11). Para este caso, el conocimiento que se construye y legitima en las prácticas de cuidado no médico que describieron los participantes.

Se retoma también la invitación que Maturana hace sobre el 'lenguajear' como constitutivo del sentido de lo humano, que, para el caso de la memoria, remite a esa posibilidad de interacción y subjetividad con aquellos que narran su historia, pero también su presente (13). Esa memoria viva de sus cuidados, en relación con una enfermedad que ha supuesto inventarse maneras particulares de estar en un cuerpo que pierde sensibilidad y que debe cuidarse. Que ha requerido transacciones y negociaciones, ensayos y errores para desafiar esas heridas que permanecen, a pesar del dictamen médico de que se es un "curado social”.

Esa manera particular de cuidarse devela, como lo enuncian Scheper-Hughes y Lock, relaciones entre significados y estructuras: una memoria que permite comprender el carácter simbólico e interpretativo de las transacciones que se dan en el acto del cuidado del paciente con lepra y quién se relaciona con intervenir en él (14).

Este recorrido incluye también las dimensiones éticas y políticas del cuidado que reconocen en este la responsabilidad social sobre el bienestar colectivo y permiten comprender que, en cualquier acto de cuidar, hay una decisión moral que tiene consecuencias para la vida. Que, además, en este intercambio intersubjetivo se requiere respetar y validar las 
particularidades que implica cuidar a alguien en su contexto, reconociendo su voz, su singularidad y sus intereses. En este sentido, esa memoria habla de sentidos e intereses que se comparten, se transan, se transforman, se transmiten y, también, se modifican, se recrean y, algunos incluso, se desechan y se olvidan (15-17).

La información fue recabada de diversas fuentes de información, como testimonios de pacientes, auxiliares de enfermería retiradas y activas, médicos, trabajadores del sanatorio que han desempeñado diversos oficios y otros pobladores, como expendedores de yerbas, boticarios y comadronas; además, observación participante, visitas domiciliarias, revisión de archivo del sanatorio y privado de personas interesadas y preocupadas por la historia de la lepra. El trabajo de campo se realizó entre septiembre de 2015 y noviembre de 2016, con visitas de periodicidad mensual al territorio. En todos los casos se contó con el asentimiento informado de los participantes para grabar las entrevistas y para usar las citas que aparecen en el texto, se marcan con iniciales para guardar confidencialidad, como parte de las consideraciones éticas. La información se consignó en audios que fueron transcritos, diarios de campo y fotografías. Una vez ordenada la información, se realizó un análisis de contenido, que fue discutido y triangulado con los participantes, investigadores y estudiantes que formaron parte del equipo de investigación y algunas fuentes teóricas relacionadas. Estos resultados son los que se presentan a continuación.

\section{Desarrollo}

T os hallazgos de la investigación permiten proponer dos expresiones de los cuidados no médicos de la lepra, una signada por las relaciones que se tejen entre los saberes de quienes se desempeñaron como enfermeras y las personas que los reciben, que aparecen bajo la categoría 'Entre la mística del cuidado y el poder del saber biomédico'. Y una segunda que reúne los hallazgos que muestran las búsquedas de los pacientes que se acercan a prácticas no médicas, intermediadas por otros agentes con saberes 'autorizados' legitimados entre los pobladores por la creencia en su efectividad, como los curanderos, boticarios, o expendedores de yerbas medicinales que corresponde a la categoría 'Naturaleza, confianza y solidaridad'; búsquedas que se concretan en expresiones de cuidado a sí mismos, entre sí y a su entorno (11-12).

\section{Entre la mística del cuidado y el poder del saber biomédico}

Este primer grupo de expresiones de cuidado está intermediado por quienes se denominan los agentes del saber biomédico. En la propuesta de Menéndez, estos agentes hacen parte del Modelo Médico Hegemónico, por medio del cual la biomedicina —-particularmente, pero 
no solo la alopática - configura un tipo de atención en la que se establece una relación de subalternidad entre quien brinda la atención y quien la recibe (7). Los hallazgos mostraron que, si bien en algunos de sus procesos de atención, estos agentes hacen uso de este poder que les permite subalternizar a los sujetos de cuidado, también expresan, en otros de estos, unas disposiciones particulares relacionadas con su capacidad de escucha, de observación, de paciencia y de trascendencia, que se acompaña de un posicionamiento y negociación en el territorio de manera cotidiana. Pues, como lo enuncia Platarrueda en su estudio sobre consensos y disensos sobre la lepra: "algunos empleados de los sanatorios suman a su experiencia en salud la de ser descendientes de enfermos, o de haber vivido la enfermedad ellos mismos" (18). Estas expresiones, que en términos terapéuticos corresponderían a esto que el campo mostró como mística del cuidado, son en la mayoría de casos en los cánones del modelo médico hegemónico, subvaloradas tanto en la formación como en el desempeño profesional. Las siguientes narrativas, expresas en apartados, son muestra de esta tensión.

\section{Más allá de la atención corporal}

Es claro ver como la atención incluye dimensiones humanas donde los elementos espirituales cobran un interés particular. En una de las entrevistas se menciona que "las hermanas eran las jefes en enfermería, eran las anestesiólogas, eran las enfermeras que acompañaban al médico en cirugía. Pero alrededor de ese instrumental estaba la cuestión espiritual” (comunicación personal con HR, 2015 oct).

Así, hay una comprensión de la dimensión espiritual que no se da únicamente por la presencia de religiosas en el servicio de enfermeras, sino que es recordada entre las mujeres del San Vicente como algo tan necesario como las curaciones o el alimento, como un ritual: "Anunciamos por el micrófono, siempre que hay misas martes y domingos, yo invito a las personas a que vengan, porque el valor que tiene una misa no lo tiene todo el dinero del mundo" (comunicación personal con N, 2015 oct).

\section{Observación, vocación, mística del cuidado}

El cuidado es un proceso. Este no debe limitarse a procedimientos aislados, sino que requiere dedicación, mística, entrega. Así, lo expresó una de las entrevistadas,

Me asignaron además el manejo de las úlceras, el programa de curación ¿qué había en el programa de curación? la enfermera, la auxiliar de enfermería que estaba en el servicio, cualquiera cogía el programa, lo hacían más como una actividad rutinaria: bañar, dar medicamentos, curar, tendido de cama, esas eran las actividades, o sea, todos los días el paciente tenía una enfermera diferente en el programa. (Comunicación personal con HR, 2015 oct) 
Entonces se empezaron a mirar una serie de cosas y de complicaciones en la curación del paciente más allá de las aproximaciones médicas,

Por ejemplo, hay cosas que afectan [...]. No es creencia mía sino, por ejemplo, la enfermera que llegue trasnochada o bebida, eso es fijo que se refleja en la úlcera del paciente, lo vi [...] por ejemplo, los estados de ánimo de las personas, el temperamento, las relaciones sexuales, que la muchacha el día anterior tenga relaciones sexuales con su pareja, llegue al momento de la curación pueden ser tabúes, pueden ser mitos, leyendas, pueden ser lo que sea, pero lo veíamos reflejado ahí todo esto, veíamos nosotros que iba afectando y que iban desmejorando las úlceras del paciente”. (Comunicación personal con HR, 2015 oct)

Desde esa comprensión de la enfermedad, retomando las narrativas de las personas, se empezaron a integrar en la atención otros elementos como "la parte humana, psicológica, social de las personas” y se estableció un camino que conducía a capacitar sobre esta nueva forma de interacción (comunicación personal con HR, 2015 oct).

Se determinó organizar un grupo de personas que fueran exclusivamente para realizar esto, que empezáramos a capacitarlas para que no fuera un procedimiento más, sino que fuera un grupo específico con una serie de características, unos perfiles. Primero que todo, una persona empática que tenga muy buena relación, el paciente se cura más a veces con el 'buenos días', que con el iponga el pie que lo voy a curar y yo sé mucho y usted no sabe nada; segundo, una persona equilibrada psicológica y afectivamente estable; otra de las características que la persona fuera de un temperamento no tontica, pero sí que fuera una persona... ¿cómo podremos decir? tranquila, serena. Otra de las cosas que veíamos era, pues, que le gustara, eso era básico para que realizara este procedimiento. Otra última, quizá la poníamos de última, que la persona supiera hacer el procedimiento, porque yo le puedo enseñar, pero lo otro no. (Comunicación personal con HR, 2015 oct)

\section{Un trabajo paso a paso}

Requirió paciencia consolidar no solo el equipo de trabajo sino maneras de hacer las cosas, ir mostrando con perseverancia que se podía mejorar.

No existía esterilización en este hospital, pues existía una sala de material que era en la unidad de consulta externa que era un hueco porque era una pieza que había sobrado en donde tenían cosas para reciclar. Entonces yo empecé como a tramitar la cosa porque tampoco era coherente, yo estuve haciendo un material estéril en medio de este mugrero. Ahí trabajamos como un mes, después se empezó a tramitar la central de esterilización. Nosotros pasamos de entregar en bolsas de kilo, por ejemplo, usted tiene una úlcera de $3 \times 4$ entonces yo le voy a entregar 3 metros de gasa y usted en la casa lo corta y se cura, luego un material estéril, lo hacían en los servicios de enfermería y se esterilizaban en las famosas ollitas que se hacían al vapor y se envolvía en tela, después se pasó al papel craft y después al sintético, que es el que está ahorita. Entonces, en toda esa transición, se veían como los choques de las personas, el malestar que existía, el de pasar que me den a 
mí 4, 6, 12 metros de gasa según tamaño de la herida, a dármela ya cortada envuelta en papel craft. Eso le generaba al paciente una inseguridad de que no le alcanzara, él en la casa la cortaba, haciéndola rendir. Se generaban las peleas, hasta de venir amenazas y de garrote en mano a pegarnos, entonces esas transiciones generaban mucha violencia en ellos y era normal, para mí era normal porque ellos venían acostumbrados a que les quitaban muchas cosas, desde su niñez le quitaron hasta su familia. (Comunicación personal con HR, 2015 oct)

\section{El cuidado: "un trabajo muy sencillo, muy sencillo, pero a la vez muy delicado"}

El cuidar implica un posicionamiento moral y político, tomar conciencia de la justicia y la equidad.

Cuando llegué, me llamaron, me delegaron un trabajo muy sencillo, muy sencillo, pero a la vez muy delicado. En el albergue Boyacá existían las celdas para tratar a los enfermos más limitados que había, se llama el servicio de Rosario, ellos no dormían en cama y colchones común y corriente sino en muro. La tarea que se me dio fue a los hombres completos pasarlos para el Rosario y a los viejitos que estaban limitados pasarlos al San José. Porque quería que el cuidado fuera más delicado y más fácil de manejarlos, esas celdas que eran muy húmedas, muy sucias, no teníamos a veces los recursos del agua, a ellos los bañaban con manguera en su mismo cuartico, no los sacaban. El paciente permanecía ahí, entonces él quería dignificar un poquito la vida de esas personas que estaban en ese lugar. Lógicamente que la parte social mía, que la parte como enfermera fue bastante dura, porque empezaron a perseguirme, a agredirme física y verbalmente, alguno de ellos alguna vez me persiguió con un cuchillo porque yo los había trasladado. (Comunicación personal con HR, 2015 oct)

Estas narrativas dan cuenta de esa mística del cuidado, y coinciden con lo que el grupo de investigación Aurora Mas, de la Universidad de Zaragoza, denomina cuidados invisibles, definidos por Isabel Huércanos como

Aquellas acciones profesionales y autónomas de cuidado enfermero que en su mayoría resultan intangibles pero que contribuyen al bienestar y mejoría de las personas tanto o más que las acciones técnicas y delegadas dirigidas al plano únicamente físico de la persona. (15)

Estos cuidados invisibles se mantienen muchas veces ocultos por falta de relevancia o por considerarlo en algunas ocasiones “cuestiones vanas, carentes de importancia” (15).

Son expresiones de cuidados ejercidas por personal de salud, que no desconocen el valor de la biomedicina, pero que la retan cuando demuestran que cuidar requiere de sutilezas que no se evidencian en las ordenes médicas, que no se registran en los documentos oficiales, como la historia clínica, pero que cuando se trata de pensar en decidir sobre dignificar 
a las personas y brindar cuidados de calidad se hacen imprescindibles. Ponen en juego, además, la dimensión moral y política del cuidado que implica tomar decisiones a partir de ese conocimiento que se adquiere con la observación meticulosa, mística, de los detalles que hacen la diferencia. Que constituyen, como lo nombra Carmen de la Cuesta, esa dimensión artesanal del cuidado.

De otro lado, surgieron otras dimensiones relacionadas con el poder que se confiere socialmente a ese saber autorizado, por la capacidad que al ejercerlo tiene de salvar barreras burocráticas, o también cuyas decisiones son interpretadas como abusos de este que generan inequidades entre los sujetos de cuidado, como lo muestran los siguientes relatos, en los que se diferencia el lugar del poder y la estratificación del cuidado.

\section{El poder del saber autorizado}

La semana pasada, yo hice una valoración y le di la orden al señor y llamé por teléfono al lugar donde le tenían que entregar el para su curación y me fui. Cuando yo llego, a la hora y media, lo tenían dando vueltas por el hospital; buscándome para que les mandara el papelito. Me dio una rabia cuando yo veo al viejo en las escaleras y yo ¿mi viejo, sumercé no tenía afán de irse? ya estaba bravo. Les dije "No, no es posible, esto no puede suceder, ustedes tienen que atender, el procedimiento es que está o por teléfono, verbalmente, o por escrito”. Entonces claro, ¡la jefe está brava, la jefe está brava! qué cuento que esta bravo o que está contenta, a mí no me interesa, no les interese lo que vean en mi cara, interese en lo que vean en el paciente. (Comunicación personal con HR, 2015 oct)

\section{La estratificación en el cuidado. En San Vicente: playa alta, playa media y playa baja}

En el Boyacá [albergue de hombres]. En los salones de arriba servicio San José, estaban los pacientes que podían salir, que podían hablar, que podían quejarse; toda la parte social que podían hacer las tenían en los salones de arriba y allí en el rinconcito de abajo en el servicio Rosario tenían en esas celdas a los pacientes más malitos y más delicados de la institución, allí tenían a los pacientes con problema mental, con la discapacidad más grande, o sea, como tratando de esconderlos, de edad avanzada y personas muy malitas, muy mutiladitas. El pabellón Saenz [en el albergue san Vicente de Mujeres] ¿Hay alguna característica especial de las personas que están en el pabellón? Pues aquí no hay ninguna especial, mija. Todas somos iguales y estamos acá por el mismo motivo, por la enfermedad de Hansen. (Comunicación personal con N, 2016 oct)

Sin embargo, al indagar por este mismo lugar a una paciente de otro pabellón dice: "aquí es como en el concurso de televisión: playa alta, playa media y playa baja, nos tienen estratificadas” (comunicación personal con PP, 2016 oct).

La dos anteriores narrativas testimonian decisiones que han permitido una estratificación, especialmente en los albergues, donde, bajo criterios del personal de salud que administra y ordena sobretodo la disposición de pacientes, se asignan locaciones con algunas 
condiciones que favorecen la privacidad y la autonomía de quienes tienen una mayor funcionalidad y no requieren asistencia directa. Estas decisiones, que corresponden no solo a lógicas biomédicas (por ejemplo, pensar en el grado de funcionalidad de las personas), sino al bienestar de las personas con menor autonomía (cuartos con atención permanente de personal de enfermería), son leídas como privilegios que estratifican el cuidado.

La situación en la cual no se atiende a la persona, a pesar de la orden de alguien que encarna poder como trabajador de salud, da cuenta también de cómo el poder del saber biomédico es desafiado.

Estos relatos evidencian que para comprender las relaciones de cuidado se requiere profundizar en la tensión entre intereses, actores involucrados, saber el significado de la palabra ‘cuidado' y las estructuras que lo permean (14).

\section{Naturaleza, confianza y solidaridad}

Bajo esta categoría se agrupan las alternativas de cuidado no médico que se rastrearon, que incluyen las búsquedas de los pacientes, intermediadas por otros agentes con saberes autorizados y las expresiones de cuidado que ellos se procuran a sí mismos, entre sí y a su entorno. La memoria a este respecto resultó amplia y variopinta. Se ubicaron por lo menos tres saberes importantes a este respecto. El primero recoge una amplia farmacopea relacionada con el uso de plantas medicinales, el segundo agrupa las referencias a curanderos con reconocida trayectoria en el pueblo y, en especial, uno que certifica tratamiento de comprobada eficacia y cura de la lepra y un tercero que da cuenta de prácticas solidarias de tutoría entre pacientes y otras ocupaciones de cuidado.

\section{Las yerbas santas, las mezclas y sus usos}

La información respecto del uso de las plantas y mezclas proviene en su totalidad de las entrevistas realizadas a pacientes, enfermeras y curanderos (personas reconocidas entre la comunidad por sus poderes y conocimiento con capacidad de curar). Las referencias ubican algunas plantas de manera singular a las cuales atribuyen poderes curativos para una o varias afecciones. Hay también combinaciones de plantas para potenciar efectos terapéuticos y, en otros casos, la mezcla de sustancias diversas contribuye al amplio recetario usado.

Ordenar este recetario no resultó fácil; después de varios análisis, se describen tal y como las reseñaron, en la mayoría de casos, relacionándolas con la afección a la cual estaban 
destinadas. Dichas afecciones corresponden a las molestias más frecuentes causadas por la enfermedad, de acuerdo con el curso que sigue en cada caso particular.

Tabla 1. Afecciones más frecuentes con las correspondientes yerbas y mezclas usadas para su manejo

\begin{tabular}{cc} 
Afecciones & Yerbas usadas \\
\hline Dolor & $\begin{array}{c}\text { Marihuana: mezclada con alcohol y aplicada tópicamente calma el dolor. } \\
\text { Quita todo: quita el dolor y desinflama las heridas. } \\
\text { Nim: en baños disminuye el dolor. }\end{array}$ \\
\hline Erisipela & Benzetacil: se saca el polvo del frasco y se aplica sobre la piel. \\
\hline & Agua hoja de papayo y hoja de guanábano: baños a los pacientes para desinflamar, "sirve para todo". \\
Cajeto: sirve para desinflamar, que la herida no supure y reduce el dolor. \\
El Nacedero: ayuda a desinflamar. \\
Quita todo: planta que quita el dolor y desinflama las heridas. \\
Nim: baños con esta planta, ya que disminuye la inflamación.
\end{tabular}

Barra de jabón pino amarillo, más panela: hace una bolita, cuando pica en el sitio y se la pone y eso saca todo lo que recoge.

El Nacedero: para tratamiento de granos.

Alcanfor y alcohol: cuando los perforantes destruyen los huesos, la mezcla de alcanfor y alcohol en una mecha (algodón torcido), se usa para sacar las astillas (forma en la que salen los huesos) y así se cura el perforante.

Perforantes plantares

o chumeros

Cremas para perforantes: cremas de Yodosalil, polvos de metronidazol, Baycuten y la panela porque saca el agua. La panela se dejó de usar por el clima, ya que por el calor el dulce llama las hormigas.

Otras cremas usadas comúnmente por los pacientes: Yodora, Hinds, Nivea. Vaselina: para el nacimiento de tejido en los perforantes

Reacciones lepróticas
Guasca de plátano y Mata ratón: en las reacciones lepróticas, era común que envolvieran a los pacientes en hojas de guasca de plátano y los bañaran con mata ratón.

Mata ratón: "cuando a su mamá le daban rebotes de nerviosa", le ponían una cama de solo mata ratón y la acostaban ahí. Igualmente, se hacían plasmas de mata ratón para bajarle la fiebre. Sábila: licuar sus cristales y colar, poner para tratamiento de pacientes con lepra nerviosa. 
Anamú: se usaba para tratar las úlceras, pero le atribuyeron como efecto secundario disminución de la visión y se desestimuló su uso.

Agua hirviendo: pacientes que tengan piel sensible o muy blanca, sustituir la solución salina por agua hervida, ya que la sal del suero hace que la piel se les queme. Realizar estos baños cada 8 días, lavando muy bien las heridas y lubricando la piel.

Acetato de aluminio con crema Lubriderm o con vaselina: esta mezcla se hace para lubricar la piel después del baño con agua hervida. Se puede con la Lubriderm o con vaselina, se hace la mezcla con el acetato de aluminio, esto se hace en el caso que la herida no este infectada. Ácido muriático: algunas pacientes se aplican este ácido para las úlceras.

Baño de gasolina con piedra pómez: "un paciente que se baña con gasolina y piedra pómez para sanar las heridas. Este baño sí funciona, pero quema la piel de alrededor”.

Úlceras y heridas de la piel

Caléndula, mosquero y árnica: para las úlceras, bañar con agua de caléndula, mosquero y árnica. "La caléndula es buena para todo".

Cogollo de guanábana y de mango: baño de heridas y úlceras.

El Nacedero: ayuda a que no le supuren las heridas.

Domeboro: agua con acetato de aluminio, mezcla para curación a personas con heridas infectadas.

Fórmula del joven médico de la universidad de Cartagena Hugo Corrales Lugo para úlceras:

solución alcohólica de violeta de genciana al $1 \%$ (30 gramos), solución de nitrato de plata al $1 \%$ (30 gramos), solución de Tanino al $15 \%$ (30 gramos).

Gelatina sin sabor: Rafael Suárez cuenta que las personas mezclaban gelatina sin sabor con pastas para curar las heridas.

Guayaba: su pulpa espesa y colada la ponían sobre las úlceras.

Gulupa: rompen la gulupa, la trituran dentro de la cáscara y con ese jugo mojan la gasa y se la ponen en las heridas.

Hoja de plátano con mantequilla: los enfermos de lepra eran acostados sobre hojas de plátano con mantequilla para que las sábanas no se pegaran a las heridas.

Mezcla de cefradina con caléndula y sábila: para las úlceras.

Mezcla de dermatomicol con ácido fólico: "para rellenar y llamar la piel, después de que las úlceras ya se han limpiado con el manejo con caléndula y lavado con jabón rey azul”.

Mezcla de Metronidazol con gelatina: mezclar con gelatina sin sabor para las úlceras.

Úlceras y Mezcla de Sulfadiatina más limón: moler bien las pastas, exprimir 3 limones y con ese polvo

heridas hacer una pasta. Sirve para curar las úlceras (María Sanjuan).

de la piel Nim: su agua tiene efectos terapéuticos parecidos a la caléndula para limpiar úlceras, preparada con mezcla de azúcar y sal en la proporción de un suero.

Sarnol: Mezcla realizada por Florentino Camacho, "destinada para curar el mortificante efecto de rasquiña producido por la causa crónica de la enfermedad de la lepra” (19).

Tetraciclina y ampicilina: antibióticos para limpiar no tomadas sino aplicadas localmente. Yodo: "se aplicaban yodo para curarse las heridas".

Yuca: raspada en las úlceras a un paciente, ayuda a la limpieza y desinfección de úlceras.

Úlceras

gástricas medicamentosas
Papa pelada: tomar el zumo de la cáscara pana sanar las úlceras gástricas producidas por los medicamentos.

Además, entre las recomendaciones estaba no consumir los siguientes alimentos, ya que generan reacciones específicas en personas enfermas de Hansen: papa criolla, yuca, pescado, aguacate, chicharrón, carne de marrano, grasa, evitar fritos y fajarse bien.

Estas prácticas han estado intermediadas por personal del Sanatorio, dadas las condiciones de salud que en algunos casos impiden la movilización de los pacientes, como consta en este testimonio: 
Cuando yo era mensajero de los albergues, pasaba todos los días por las habitaciones y los pacientes me encargaban; tráigame dos pastillas de alcanfor, a mí un sobre de esas para los hongos, unas hojas de Nim para preparar el agua y regresaba después entregando los encargos y las vueltas. (Comunicación personal con G, 2015 jun)

E implican también esas acciones del cuidado invisible por parte del personal de salud, que se mencionaban en el primer apartado, representado en este caso por una negociación permanente:

Uno no puede llegar a pelear con el paciente y decirle "no se eche eso que no sirve para nada”, tiene que negociarle y decirle bueno, probemos a ver si con esa gasita untada de gulupa, como usted, dice mejora, pero si en dos días no está bien, volvemos al otro tratamiento. (Comunicación personal con AE, 2015 oct)

\section{Curanderos reconocidos y otros tratamientos}

En este territorio confinado, a pesar de la política segregacionista, había intercambio de pobladores foráneos con los habitantes que eran principalmente los pacientes, algunos familiares y quienes representaban el estamento médico y religioso. El nutrido número de curanderos da cuenta de lo prolijo de este intercambio y los testimonios de la confianza que en algunos de ellos se alcanzó a tener:

- Sr. Bullén: entre 1880 y 1896 algunos enfermos estuvieron recentándose con un remedio que traía este señor, el cual ponía una sola gota en una botella de agua que debía tomarse el paciente en tres o cuatro días (20).

- Florentino Arias: el tratamiento consistía en enterrar al enfermo hasta el cuello en una especie de sepultura por un corto tiempo, y en esta posición se les suministraba en brebaje; 2 o 3 bienaventurados se sometieron a este bárbaro sistema, retirándose el curandero por falta de clientela (20).

- Antonio Buitrago: desde el Quindío anunció la curación de la lepra. Muchos enfermos se vieron esperanzados y se trasladaron allí, sin tener en cuenta las medidas de aislamiento, más de 30 enfermos se trasladaron. Dos o tres meses duraron en tan especial sanatorio, y uno a uno fueron regresando al Lazareto en peores condiciones de enfermedad y de bolsillo (20).

- Ángel García: cubano, conocido como curador de la lepra. Llega al Lazareto en 1918 con el fin de aplicar el tratamiento a los enfermos, después de aplicarlo emprendió vuelo sin ver los efectos de su tratamiento (20).

- Sor Marcelina de la Cruz, Sorcita: fue una hermana de los sagrados corazones, enferma de lepra, que manejó una botica en el pueblo. Cuentan que preparaba un remedio llamado “Caraña”, que servía para los orzuelos, se ponía en el ombligo y hacia que estos 
se secaran, también preparaba una loción para los hongos (comunicación personal con MTR, 2015 nov).

- Dr. Mauricio Méndez: antiguo médico del sanatorio y alcalde popular en una ocasión, hoy médico del policlínico que corresponde al sns Cundinamarca y consulta particular de homeopatía, con amplia demanda. Esta terapéutica aparece que se usó desde los primeros años del lazareto por el Dr. Marcelino Liévano y, continuando en los años siguientes por los Drs. Luis Carlos Padilla, Amalia Liévano, Crisóstomo Bautista, Enrique Aguilera y José Correal. Miembro honorario del Instituto Hanemann en Colombia, y otros aficionados, ha conquistado muchos devotos por los excelentes resultados que los enfermos han obtenido con esta medicación (20).

- Hermana Edelmira: de la orden de las Hijas de los Sagrados Corazones de Jesús y María. Medicina restaurativa, iridiología (comunicación personal con HE, 2015, nov).

También hubo quienes ofrecieron tratamientos integrales, que incluso certificaron la curación de la enfermedad, como en el caso de Florentino Camacho González, quien recomendaba el tratamiento de masajes cuyo objeto era "remover depósitos de sangre y líquidos estancados: disolver tubérculos y lepromas; kistes e induraciones, descongestionar los sistemas nervioso, muscular y digestivo, activando sus funciones”. Además, se agregaba "El raspado", que tenía por objeto "extraer del cuerpo del enfermo: el vello, las escamas, los callos y la piel muerta, consiguiendo por este medio: (la ejecución del masaje y la Extracción de la piel muerta), la circulación de los líquidos estancados, destinados por la naturaleza a dar vida a cada uno de los miembros atrofiados del Enfermo. Consiguiendo además la exhalación del sudor, la devolución de la sensibilidad, la elasticidad de la piel y de los demás miembros afectados". Además de lo que Florentino nombra como "pequeña cirugía”, que tiene por objeto, extraer del cuerpo del enfermo, los residuos de los tubérculos, lepromas, kistes, enduraciones y líquidos que no hayan sido asimilados ni eliminados por medio del masaje.

Adicionalmente, el enfermo debía realizar por su cuenta lo siguiente:

1. Baño diario

2. Inmediatamente después del baño, una fricción de aceite "untura"

3. Tomar agua hervida y caliente

4. Hacer ejercicio al aire libre

5. La gimnasia

6. La excursión

7. El purgante (sulfato de magnesia cada ocho días, tres cucharadas grandes) 
8. Hasta que el estómago defeque 2 veces diarias

9. Una cucharada grande, todas las veces que se demore la defecación más de las 24 horas. Hasta obtener una defecación normal (o dos diarias)

10. La excursión

11. Una vida honesta y buena alimentación

Este "curandero" afirma al final de su escrito lo siguiente:

Muchos de uds. probablemente se escandalizan al oírme hablar, o al leer estos renglones, sobre la manera como he llegado a descubrir la curación de la enfermedad de la lepra. Convencido como estoy de esta verdad, por el éxito de mis procedimientos, no hago más que obedecer leyes de la naturaleza. Afirmar mis descubrimientos y comunicarlos a mis semejantes".

Con este tratamiento, consta en la misma fuente la curación certificada ante notario público de una de las personas a quién trató Florentino Camacho:

Hago constar por medio del presente testimonio, que, habiendo sufrido por espacio de varios años la enfermedad de la LEPRA, sin que ningún tratamiento médico me aliviara de las terribles dolencias, me sometí a un tratamiento de MASAJES del señor Don Florentino Camacho González, habiendo obtenido una curación pronta y estable, que me permite disfrutar mejor de la vida. En constancia del presente firmo: En Agua de Dios a los veinticinco días del mes de mayo, de 1960. (Jesús E. Murillo)

Las anteriores expresiones de la memoria del cuidado no médico de la lepra en Agua de Dios y su presencia en documentos escritos, pero principalmente en la memoria oral de sus habitantes, coincide con la afirmación de Menéndez sobre que "Frecuentemente desde la perspectiva del Sector Salud (ss) y de la biomedicina, toda una serie de actividades de atención a los padecimientos es negada, ignorada o marginada, pese a ser frecuentemente utilizada por diferentes sectores de la población” (7). Además de revelar lo que Perdiguero y Menéndez proponen como pluralismo médico, que hace referencia a las variadas formas de atención para diversos problemas e incluso para un mismo problema de salud, predominantes en los grupos sociales que no distinguen clase, etnia u otras diferencias entre ellos $(5,21)$.

Dan cuenta también de cómo los profesionales de salud entran en diálogo con los conocimientos de los saberes autorizados y deben negociar permanentemente entre estos y los conocimientos biomédicos, lo que implica, una vez más, una necesidad de tomar decisiones sobre el cuidado, que no solo desafían el cocimiento biomédico que no que reclaman de este personal decisiones éticas y un ejercicio político al decidir sobre qué hacer al cuidar. Las siguientes citas ilustran esto en lo que se denomina acá, el abordaje desde la solidaridad y otras ocupaciones de cuidado. 
Hacerse cargo, "la tutora legal"

Yo tuve 14 pacientes a cargo. Yo les manejaba su dinero, les compraba los pañales, todo, absolutamente lo necesario. No, es que mire, mucha gente como yo no tiene familia, no tenemos casa donde llegar, ni con quien estar, entonces para esas personas de las mismas viejitas, todas imposibilitadas, todas en silla, unas con sonda y todo eso, no podían manejar sus recursos, su ayuda que nos da el Gobierno. Entonces, el Sanatorio a través de la trabajadora social le decían, usted como es la más joven, si es capaz de hacer eso, hágalo.

¿Cómo una tutora? Exacto, yo en el Boyacá tengo a X, él me lo dio el juzgado de Girardot como psiquiátrico, yo le manejo los centavos, esos pañales que usted ve y su cajón con tres cajones llenos de ropa, todo lo que él necesita, le tengo la funeraria paga, todo. Me llamaban de Girardot cuando lo hospitalizaban, a ir a firmar, a autorizar una cirugía, que tal cosa, pero siempre me tocó llevarlos a mí solita al entierro, a la funeraria y yo irlos a enterrar. Me hago cargo de ellos hasta la muerte. (Comunicación personal con N, 2015 oct 21)

\section{Otras ocupaciones de cuidado: las plantas, las mascotas, pintar, arreglarse}

Todas esas matas que usted ve, todas esas palmas, todo eso que ve ahí en el patio, yo las he comprado, las hemos sembrado, le pago a un señor que me las siembre, que me le quite las palmas secas, yo misma las riego y tengo bonito al frente porque soy y me gusta que se vea fresco, que se vea bonito. Yo tengo perra, por orden del psicólogo debo tener una mascota, me relajo acariciándola... Yo me entretengo mucho pintando, pero tejer en dos agujas y en crochet a poco veo para meter la aguja no veo, entonces no lo hago. Eso lo hice mucho tiempo Hice blusas, manteles de punto de cruz... Me perfumo, porque me pongo mis joyas, porque me arreglo apenas me levanto, me pinto los labios y me salgo por allá. (Comunicación personal con N, 2015 oct)

Estas narrativas, que dan cuenta de las expresiones de solidaridad y otras formas de cuidado, pueden considerarse como acciones de autoatención en la acepción amplia que del concepto propone Menéndez, refiriéndose en el campo de la salud colectiva a todas las formas de autoatención que se requieren para asegurar la reproducción biosocial de los sujetos y grupos. Que incluyen "no solo la atención y prevención de los padecimientos, sino las actividades de preparación y distribución de alimentos, el aseo del hogar, del medio ambiente inmediato y del cuerpo" (7).

\section{Conclusiones}

E ste recorrido por la memoria de los cuidados no médicos de la lepra propone continuar
con el conocimiento sobre esta, respecto de investigaciones que han marcado hitos en la manera de acercarse a conocerla en el país. Una de ellas la investigación de Diana 
Obregón, titulada "Batallas contra la lepra”, que muestra la interacción entre el afán de la medicina por conocer sobre esta enigmática y bíblica enfermedad y cómo aprovecha este camino para posicionarse socialmente en Colombia. Además de permitir rastrear una visión particular de nación y la relación entre la medicina y el Estado, Obregón plantea cómo la lepra fue determinante en el proceso de ascenso de la profesión médica y, con ello, la consiguiente "pérdida de prestigio y poder de otros grupos tales como religiosos, curanderos y los mismos enfermos” (22).

Otras investigaciones corresponden a las investigaciones de Claudia Platarrueda y la de un abordaje más etnográfico: "Los mutilados del oprobio", estos estudios permiten escuchar la voz de personas con lepra y lo que implicó la manera particular de ordenamiento social que se fue tejiendo alrededor de estos sitios “confinados", con acompañamiento importante del clero, y de la mano de un Estado con una presencia magnánima en leyes y disposiciones, pero escasa en institucionalidad. Unos lugares en los que se recrearon maneras particulares de gobierno, en las cuales los médicos y los sacerdotes llegaron a ostentar más poder que los funcionarios públicos, además de la participación de los enfermos en la dinámica institucional y política de estos territorios, que transitan por representaciones que muestran el lugar "unas veces como espacio de encierro y muerte y otras como lugar de refugio y reconstitución vital” (23).

Este trabajo, al igual que otras producciones, busca contribuir a valorar el patrimonio inmaterial en que se constituye la memoria misma del cuidado y evidenciar las tensiones permanentes entre los agentes del cuidado (personal de salud, personas con saberes autorizados y pacientes) que se disputan un campo de legitimidad (8-10). El recorrido muestra una constante negociación de saberes y un reto a la propuesta del modelo biomédico que pretende erigirse como hegemónico, pero que es reconfigurado todo el tiempo incluso por sus mismos representantes, cuando evidencian por ejemplo el uso de yerbas para las curaciones de los pacientes, que van siendo incorporadas incluso como "protocolos" de manejo de heridas. Situaciones y transacciones de cuidado que hacen que el personal de salud comprenda y valore ese saber autorizado como parte legítima de las manifestaciones de cuidado en el territorio $(11,12)$. Lo cual deslocaliza el cuidado de la injerencia delegada al personal de salud y pone de manifiesto esa pretendida hegemonía del modelo médico que intenta la exclusión de otros modelos de atención; esto lo que permite es que se transformen y apropien en la práctica y deba reconocerse hoy más que su conflictividad, su complementariedad (7).

La memoria del cuidado se torna trascendente, como lo enuncia Laza:

Por la imperiosa necesidad, en un país multicultural como lo es Colombia, de acercarse, comprender y respetar la "mirada del otro", del no profesional acerca de cómo se cuida y como este se sustenta en una "visión" particular del mundo diferente a la del sistema biomédico. (24) 
Lo anterior, por la capacidad de la memoria colectiva de permitir a los pueblos actualizar el presente y maniobrar con el pasado en su cotidianidad, como forma de desarrollar "un empoderamiento real y no impuesto por otros [...] en el cual las categorizaciones e identidades hegemónicas entran en constante revisión, recomposición y negociación” (24).

Estas múltiples alternativas de cuidado que se tornan colectivas evidencian que

La subjetividad en la enfermedad y del manejo de la misma por el individuo deriva la búsqueda de nuevas alternativas y terapias para dar respuesta a las necesidades y no solo la cura de la enfermedad, y que a través de la biomedicina no ha sido encontrada. (25)

Lo anterior concuerda también con la crítica que hace Platarrueda respecto de los manejos biomédicos de la enfermedad que son controvertidos en muchos casos, especialmente, en lo que refiere "a las nociones de contagio, enfermedad y curación” (18).

Este pluralismo de la memoria del cuidado no médico de la lepra permite también interpelarlo desde la propuesta de Guilligan, citada por Alvarado, de la ética del cuidado, que reconoce la responsabilidad social desde donde se busca el bienestar colectivo basado en decisiones morales que tiene consecuencias para la vida presente y futura (14). Aboga por reconocer las diferencias y particularidades que implica el cuidado del otro en su contexto, atendiendo a la benevolencia como centro de las relaciones sociales y de la ética del cuidado, plantea la necesidad de reconocer lo que denomina una segunda voz, en el sentido de reconocer al otro en su particularidad $(13,14,16)$.

Los hallazgos amplían la mirada del cuidado propuesta desde la biomedicina como acciones realizadas por el personal de salud y permite compartir con Collier la afirmación de que el cuidado es todo lo que permite "mantener la vida asegurando la satisfacción de un conjunto de necesidades indispensables para la vida, pero que son diversos en sus manifestación” (17) Además, "forjan formas de hacer y costumbres, y se erigen en un patrimonio de ritos y creencias de un grupo" (24).

Este acercamiento etnográfico a la memoria de los cuidados no médicos de la lepra en la voz de los habitantes de Agua de Dios reconoce, como lo propone la Antropología médica, "que todo el conocimiento relacionado con el cuerpo, la salud y la enfermedad son culturalmente construidos, negociados y renegociados en un proceso dinámico a través del tiempo y el espacio” (26, 27). Esto evidencia la necesidad que plantea Menéndez:

Si el sector salud quiere conocer o implementar el sistema de atención real que utilizan los sujetos y conjuntos sociales, debería identificar, describir y analizar las diferentes formas de atención que los conjuntos sociales manejan respecto de la variedad de padeceres reales e imaginarios que reconocen como afectando su salud. Por supuesto que esto no supone que reduzcamos la identificación de los padeceres ni de las formas de atención solo a los que reconocen los conjuntos sociales, sino que este es el punto de partida para establecer la existencia de los padeceres y de las formas de atención que los grupos no solo reconocen, sino que sobre todo utilizan. (7) 
A partir de los elementos expuestos, puede concluirse que la memoria de los cuidados no médicos de la lepra en Agua de Dios muestra cómo la ciencia y su saber, que buscan erguirse como hegemónicos, han sido siempre retados por prácticas que subvierten este llamado "conocimiento biomédico". A su vez, este se recrea con la presencia de esos otros "saberes autorizados”, mediante prácticas cotidianas mediadas por transacciones que enuncian otras situalidades y capacidades de agencia de los sujetos.

Así, en este texto es posible dar cuenta de otros elementos del cuidado que no suelen estar visibilizados en los discursos académicos y rutinarios de lo que se enuncia como un cuidado profesional, para el cual se prepara al "personal de salud", que se refleja en esa necesidad de la mística, la paciencia, la escucha y la observación, además de la capacidad de negociación. En este estudio es necesario plantear el reto que supone hoy en día para este recurso humano integrar estos elementos de los saberes autorizados y saberes legos que se gestionan todo el tiempo y se legitiman en los procesos de cuidado, más allá de las instituciones hospitalarias y los cánones de la biomedicina.

Los testimonios ilustran esa dimensión compleja de quienes, en un territorio particular como lo es Agua de Dios, signado por la lepra, recrean la importancia de la dimensión espiritual del cuidado, otorgando un lugar privilegiado a la presencia religiosa en el ámbito del cuidado directo de los pacientes y su desmantelamiento deja una estela de su descuido y abandono.

En la misma línea, debe darse valor a la amplia farmacopea herbolaria reunida por medio de testimonios orales de los habitantes de Agua de Dios (pacientes, curanderos, expendedores de yerbas). Esta da cuenta del valor de la memoria y la tradición oral en el cuidado y curación de afecciones, no solo las más frecuentes en los pacientes de Lepra sino otras de presencia común en cualquier ser humano, que, al ser comprada con publicaciones oficiales reconocidas como científicas, coinciden perfectamente. Es decir, se muestra cómo esos saberes, muchas veces satanizados por el "saber biomédico", son reconocidos hoy por estudios desde las ciencias naturales y otras ramas de la medicina no alopática como "científicos”. Así, recomendaciones como las de Florentino Camacho evidencian que hay un nivel de sabiduría en ese saber ancestral ligado al conocimiento y práctica de reglas que él denomina del orden natural, cuando enuncia, "todas estas prácticas que recomiendo no obedecen a otra cosa que, a las leyes naturales, vivir en consonancia con ellas”. Hoy se encuentra cómo los discursos del bienestar y de los cuerpos saludables pujan por posicionarlas como las mejores alternativas de cuidado y erigen a su alrededor todo un mercado de consumo para los llamados estilos de vida saludables. Todas estas expresiones de cuidado desafían los discursos y prácticas canónicas de la biomedicina alrededor de la lepra. 


\section{Agradecimientos}

los habitantes de Agua de Dios, especialmente a los pacientes que abrieron sus puertas - y su corazón para que esta investigación fuera posible. A la Universidad Javeriana, sede Bogotá, por el apoyo financiero y logístico, al igual que al Sanatorio de Agua de Dios. A María Alejandra Gómez Rojas, estudiante de Antropología de la Universidad Javeriana, quien en su rol de asistente de investigación apoyó de manera importante este trabajo.

\section{Descargos de responsabilidad}

$\mathrm{L}$

os contenidos del presente escrito son de responsabilidad única de su autora. El proyecto fue financiado con recursos de la Dirección de Investigaciones de la Pontificia Universidad Javeriana, sede Bogotá, y el Sanatorio de Agua de Dios E.s.e. La autora de este trabajo asume la responsabilidad de lo publicado, y la Revista de Ciencias de la Salud no asume ninguna responsabilidad por esto.

\section{Referencias}

1. Instituto Nacional de Salud. Informe del evento lepra periodo epidemiológico XIII Colombia 2017 [internet] 2017 [citado 2017 feb 12]. Disponible en: http://www.ins.gov. co/buscador-eventos/Informesdeevento/LEPRA\%20PE\%20XIII\%202017.pdf

2. El Tiempo. Vida y salud [internet]. 2017 ene 30 [citado 2017 feb 12]. Disponible en: http:// www.eltiempo.com/vida/salud/eliminacion-de-lepra-en-colombia-35640

3. Fadul F, Vargas D, Martínez M. Situación epidemiológica de lepra, Colombia, 2012 a 2014. Informe Quincenal epidemiológico nacional. Instituto Nacional de Salud 2015;20 (2):1-21.

4. Marshall C, Rossman G. Designing qualitative research. USA: SAGE Publications; 1999.

5. Galeano-Marín ME. Estrategias de investigación social cualitativa. El giro de la mirada. Medellín: La Carreta Editores; 2004.

6. Vasilachis I. La investigación cualitativa. En: I Vasilachis de Gialdino coordinadores, Las estrategias de investigación cualitativa. Barcelona: Gedisa Editorial; 2006, p23-60.

7. Menéndez E. Modelos de atención de los padecimientos: de exclusiones teóricas y articulaciones prácticas. Ciênc Saúde Coletiva, 2003;8(1):185-207. Doi: http://dx.doi.org/10.1590/ S1413-81232003000100014

8. Conrad P. Medicalization and Social Control. Annu Rev Sociol, 1992;18(1):209-32.

9. Good B. Medicina Racionalidad y experiencia. Cambridge: Cambridge University Press; 1994. 
Mística, yerbas santas y solidaridad: memoria del cuidado no médico de la lepra en Agua de Dios

10. Blasco-Fontecilla H. Humanities in Medicine: Medicalization, wish-fulfilling medicine, and disease mongering: Toward a brave new world? Rev Clin Esp (Barc). 2014;214 (2):104-7. Doi: 10.1016/j.rce.2013.08.012.

11. Brigitte J. Authoritative knowledge and its construction. En: R Davis-Floyd, C Sargent, eds. Childbirth and authoritative knowledge: Cross-cultural perspectives. Berkeley: University of California Press; 1997. p. 55-79.

12. Sargent C, Bascope G. Ways of Knowing about Birth in Three Cultures Medical. Med Anthropol Q. 1996;10(2):213-36.

13. Maturana H. El sentido de lo humano. España: Editorial Océano; 2002.

14. Scheper-Hughes N, Lock M. The Mindful Body: A Prolegomenon to Future Work in Medical Anthropology. Med Anthropol Q, 1987;1(1):16-41.

15. Bonill C. ¿Cuidados invisibles o profesionales invisibles? Arch Memoria [internet]. 2014 [citado 2016 mar 12]; 11(2). Disponible en: http://www.index-f.com/memoria/11/11200.php

16. Alvarado A. La Ética del Cuidado. Aquichan, 2004;4(4):30-9.

17. Colliére M F. Promover la vida. Madrid: Interamericana. Ed. McGraw-Hill; 1993. pp. 9-12.

18. Platarrueda-Vanegas CP. Contagio, curación y eficacia terapéutica: Disensos entre el conocimiento biomédico y el conocimiento vivencial de la lepra en Colombia. Antípoda: Revista De Antropología y Arqueología, 2008;(6):171-95.

19. Camacho F. Manuscrito Cuaderno $1^{\circ} .3^{\circ}$ Copia, 1960. [Archivo histórico del Sr. Efraín Oyaga, de Agua de Dios]. 2015 nov.

20. Gutiérrez P. Apuntamientos para la historia de Agua de Dios 1870-1920. Bogotá: Imprenta Nacional; 1925.

21. Perdiguero E. El fenómeno del pluralismo asistencial: una realidad por investigar. Gac Sanit, 2004;18(4):140-5.

22. Obregón-Torres D. Batallas contra la lepra: Estado, medicina y ciencia en Colombia. Medellín: Banco de la República y Fondo Editorial Universidad Eafit; 2002.

23. Platarrueda-Vanegas CP. La voz del proscrito; lepra y representaciones sociales de los lazaretos en Colombia. Bogotá: Universidad Nacional de Colombia; 2009.

24. Laza C. Algunos apartes del sistema teórico de la medicina tradicional. Revista Teoría y praxis investigativa, 2009;4(1):21-2.

25. Gómez-Pérez D. Palacios-Ceña D. Influencia del modelo hegemónico biomédico en la concepción y desarrollo de las terapias alternativas. Cultura de los cuidados, 2009;(25):62-68.

26. Chamorro A, Tocornal C. Prácticas de salud en las comunidades del Salar de Atacama: Hacia una etnografía médica contemporánea. Estudios Atacameños 2005;30:117-34. Doi: doi.org/10.4067/S0718-10432005000200007

27. Menéndez E. Antropología médica. Una genealogía más o menos autobiográfica. Gazeta de Antropología, 2012;28(3):8. 